\title{
The role of topic vaginal products as part of the therapeutic strategy in uterine cervix lesions
}

\author{
Nicolae BACALBASA ${ }^{1,2}$, Irina BALESCU ${ }^{3}$, Adnan Ad ALOUL ${ }^{4,5}$, Roxana BOHILTEA ${ }^{1,6}$, \\ Bogdan SOCEA ${ }^{7,8}$, Bogdan URSUT ${ }^{7,9}$, Alexandru FILIPESCU ${ }^{1,10}$ \\ ${ }^{1}$ Department of Obstetrics and Gynecology, "Carol Davila" University of Medicine and Pharmacy, \\ Bucharest, Romania \\ ${ }^{2}$ Department of Visceral Surgery, Center of Excellence in Translational Medicine, Fundeni Clinical \\ Institute, Bucharest, Romania \\ ${ }^{3}$ Department of Visceral surgery, Ponderas Academic Hospital, Bucharest, Romania \\ ${ }^{4}$ Department of Surgery, Ramnicu Sarat County Hospital, Buzau, Romania \\ ${ }^{5}$ Department of Surgery, "Titu Maiorescu" University, Bucharest, Romania \\ ${ }^{6}$ Department of Obstetrics and Gynecology, University Emergency Hospital, Bucharest, Romania \\ 7Department of Surgery, "Carol Davila" University of Medicine and Pharmacy, Bucharest, Romania \\ ${ }^{8}$ Department of Surgery, "Sf. Pantelimon" Clinical Emergency Hospital, Bucharest, Romania \\ ${ }^{9}$ Department of Surgery, "Agrippa Ionescu" Clinical Emergency Hospital, Bucharest, Romania \\ ${ }^{10}$ Department of Obstetrics and Gynecology, Elias Emergency Hospital, Bucharest, Romania
}

\begin{abstract}
It has been widely demonstrated so far that lactobacilli represent probably the most important microorganisms which are developed at the level of the vaginal cavity and which are responsible for the maintenance of an adequate environment and $\mathrm{pH}$ at this level. Once the number of these microorganisms decrease the $\mathrm{pH}$ value increase leading to the apparition of a basic medium, which favours the development of certain microorganisms such as human papilloma virus. Meanwhile it is widely known that the presence of this virus is associated with an increased risk of developing premalignant and even malignant conditions. The aim of the current paper is to investigate the correlation between the vaginal $\mathrm{pH}$, the risk of human papilloma virus infection and, in the meantime, to explore the possible benefits of local application of Cerviron, a topic, vaginal product which seems to significantly influence the risk of cervical lesions development.
\end{abstract}

Keywords: Lactobacillus, human papilloma virus, Cerviron, uterine cervix lesions

\section{INTRODUCTION}

Vaginal microbiota is characterised by the presence of a significant number of microorganisms which seem to play a central role in order to maintain an acid environment at this level; therefore, maybe the most important microorganisms which are present at this level in physiological conditions are represented by lactobacilli. These microorganisms play a crucial role in the metabolism of glycogen and it's transformation in lactic acid; it has been widely demonstrated that this chemical process is strongly influenced by the levels of oestrogens and takes place in the conditions of an anaerobic medium $(1,2)$. In this respect, whenever the oestrogen levels are modified this process is secondarily affected and the levels of lactic acid decrease; the first logical effect in such cases is the modification of 
the vaginal medium which becomes from an acid one a basic one. In these conditions, other pathological microorganisms (which, in normal conditions would be destroyed by the acid medium) will develop at the level of the vaginal cavity and will conduct to the apparition of various pathological conditions. Although the most commonly encountered such microorganisms are represented by bacteria and fungi and lead to the apparition of bacterial vaginosis or fungical vaginosis, in certain cases viral infections might develop, the most commonly encountered such pathogenic agents being represented by human papilloma virus, human immunodeficiency virus and herpes simplex virus (3).

\section{THE CORRELATION BETWEEN THE LOCAL MODIFICATIONS OF VAGINAL FLORA AND THE RISK OF GYNAECOLOGICAL CANCER DEVELOPMENT}

As mentioned before vaginal flora is comprised mainly by lactobacilli which have a protective effect on the risk of local development of different pathological conditions; however, once the number of these bacilli decrease the risk of apparition of other germs such as other bacteria, parasites, fungi or viruses increase and so does the risk of apparition of various pathological conditions. When it comes to the risk of cancer development, it is widely accepted that carcinogenesis is a complex mechanism of interactions between hormonal, immune and infectious factors $(4,5)$. Infectious factors seem to stimukate carcinogenesis process through multiple pathways: by stimulating cell proliferation, by modifying the normal functioning of the immune system, by influencing the dezoxiribonucleic acid damage and repair and by interacting with different hormones such as oestrogen (6-8). Therefore, when it comes to the risk of cervical cancer development, the most commonly incriminated germs are represented by HPV followed by other pathogenic agents such as Fusobacterium, Gardnerella vaginalis and Atopobium vaginae $(9,10)$. Another interesting correlation ship has been established between alimentary habits, vaginal microbioma and the risk of cervical intraepithelial neoplasia; therefore a diet rich in vegetables and fish seems to have a protective effect against this preneoplastic condition $(10,11)$.

\section{THE INFLUENCE OF VAGINAL MICROBIOTA ON THE FURTHER RISK OF CERVICAL CANCER DEVELOPMENT}

Although screening tests have been widely implemented worldwide in order to provide an efficient pro- gram of cervical cancer prevention and early diagnostic, in a significant number of low and medium resource countries the rate of compliance is still very low and a significant number of patients are diagnosed with this malignancy in advanced stages of the disease. In such cases a completely curable preneoplastic lesion is replaced with an advanced, aggressive gynecologic malignancy which is further associated with high rates of morbidity and cancer related death (11-14).

Meanwhile, relevant studies came to demonstrate that in patients diagnosed with preneoplastic lesions and intraepithelial lesions of the uterine cervix, HPV infection was also associated with other modifications of the vaginal flora such as a significant decrease of the proportion of Lactobacilli but also with the increase of the amount of Peptostreptococcus, Fusobacteria and Anaerococcus tetradius. Moreover, a direct proportionality relationship was observed between the number of these bacteria and the severity of the neoplastic process. Therefore, cases presenting a lower bacterial burden were rather identified to have low grade preneoplastic lesions while cases presenting increased burden rather associated high grade dysplastic lesions or even cervical intraepithelial neoplasia; moreover, a poorer long term outcome has been demonstrated in cases presenting Peptostreptococcus and Fusobacteria infections (15-17).

\section{THE INFLUENCE OF TOPIC AGENTS ON THE RISK OF CERVICAL CANCER DEVELOPMENT}

Once these aspects have been widely demonstrated, attention was focused on creating topic agents with intravaginval administrations with a higher capacity of re-establishing the normal vaginal flora. Therefore, creation of such a product which is able to re-establish a normal colonization at the level of the vaginal lining seems to be an effective weapon in order to prevent the apparition of preneoplastic and neoplastic cervical lesions. Meanwhile, association of other active principles which aim to provide a better healing of all the local microlesions which might further be considered as entering points for different species of viruses, bacteria and fungi seems to be an effective method in order to increase their benefits (18-20).

\section{THE ROLE OF CERVIRON IN PREVENTION AND TREATMENT OF PRENEOPLASTIC LESIONS OF THE UTERINE CERVIX}

Cerviron represents a topical agent which is recommended to be administrated intravaginally and which contains seven active principles. The main therapeutic 
effects of this product are related with it's antiseptic properties (due to the presence of hexylresorcinol, Thymus vulgaris extract and Hydrastis extract), to it's anti-inflammatory properties (due to the presence of calendula extract) and due to it's healing properties (due to the presence of collagen, bismuth subgallate and curcuma extract). Meanwhile, the association of bismuth subgallate seems to provide an important hemostatic effect decreasing in this way the rates of vaginal bleeding and increasing the chances for rapid healing. Meanwhile, this product seems to be particularly effective in order provide a faster healing of different inflammatory processes which, if untreated, seem to increase the chances of local malignant transformation.

As expected, maybe the most important mechanism of action through which this product can impact on the risk of preneoplastic lesions development is by modulating the compounds of the vaginal microbioma and increasing the amount of vaginal lactobacilli. Therefore, through it's antiseptic role it seems to increase the efficacy of system antiobiotic treatment and therefore to decrease the length of administration of this treatment, to decrease the risk of treatment related complications and to maximize the local antibacterian effect.
In consequence, the daily administration of this product seem to re-establish a normal equilibrium at this level and to decrease the risk of apparition of premalignant and even in situ malignant conditions at the level of the uterine cervix.

\section{CONCLUSIONS}

Local topical administration of products such as Cerviron seems to play a significant role in order to decrease the risk of development of preneoplastic conditions at the level of the uterine cervix. The main mechanisms through which cervical cancer might be prevented is by re-establishing a normal composition of the vaginal flora which can further prevent the apparition of HPV infection; meanwhile it has been demonstrated that local development of other pathogenic agents such as Peptostreptococcus and Fusobacteria can be further incriminate din the development of such pathological conditions; therefore reestablishment of the normal amount of protective microorganisms such as lactobacilli will further prevent the development of such pathogenic agents and therefore will decrease the risk of preneoplastic conditions.

Conflict of interest: none declared Financial support: none declared

\section{REFERENCES}

1. Linhares, IM, Summers PR, Larsen B, Giraldo PC, Witkin SS. Contemporary perspectives on vaginal $\mathrm{pH}$ and lactobacilli. Am J Obstet Gynecol. 2011; 204(2):120.

2. Buchta V. Vaginal microbiome. Ceska Gynekol. 2018;83(5):371-379.

3. Klatt NR, Cheu R, Birse K, Zevin AS, Perner $M$, et al. Vaginal bacteria modify HIV tenofovir microbicide efficacy in African women. Science. 2017;356:938-945.

4. Yang J, Tan Q, Fu Q, Zhou Y, et al. Gastrointestinal microbiome and breast cancer: correlations, mechanisms and potential clinical implications. Breast Cancer. 2017;24(2):220-228.

5. Weiss GA, Hennet T. Mechanisms and consequences of intestinal dysbiosis. Cell Mol Life Sci. 2017;74(16):2959-2977.

6. Perera FP. Environment and cancer: who are susceptible? Science. 1997; 278(5340):1068-1073.

7. Garrett WS. Cancer and the microbiota. Science. 2015;348(6230):80-86.

8. Koller VJ, Marian B, Stidl R, Nersesyan A, et al. Impact of lactic acid bacteria on oxidative DNA damage in human derived colon cells, Food Chem. Toxicol. 2008;46(4);1221-1229.

9. Mani S. Microbiota and breast cancer. Prog Mol Biol Transl Sci. 2017;151:217-229.
10. Champer M, Wong A, Champer J, Brito I, Messer P, Hou J, Wright JD. The role of the vaginal microbiome in gynaecological cancer. BJOG. 2018;125(3):309-315.

11. Audirac-Chalifour A, Torres-Poveda K, Bahena-Román M, et al. Cervical microbiome and cytokine profile at various stages of cervical cancer: a pilot study, PLOS One. 2016;11(4):0153274.

12. Ferlay J, Soerjomataram I, Dikshit R, Eser S, Mathers $\mathrm{C}$, et al. Cancer incidence and mortality worldwide: sources, methods and major patterns in GLOBOCAN 2012. Int $\mathrm{J}$ Cancer. 2015;136:359-86.

13. Arbyn M, Weiderpass E, Bruni L, de Sanjose S, Saraiya M, Ferlay J, Bray F. Estimates of incidence and mortality of cervical cancer in 2018: a worldwide analysis. Lancet Glob Health. 2020 Feb;8(2):e191-e203.

14. Kitchener HC, Almonte M, Thomson C, Wheeler P, et al. HPV testing in combination with liquid-based cytology in primary cervical screening (ARTISTIC): a randomised controlled trial. Lancet Oncol. 2009 Jul;10(7):672-82.

15. Castellarin M, Warren RL, Freeman JD, Dreolini L, et al. Fusobacterium nucleatum infection is prevalent in human colorectal carcinoma. Genome Res. 2012;22:299-306.
16. Yu J, Feng Q, Wong SH, Zhang D, Liang QY, et al. Metagenomic analysis of faecal microbiome as a tool towards targeted non-invasive biomarkers for colorectal cancer. Gut. 2017 Jan;66(1):70-78.

17. Mima K, Nishihara R, Qian ZR, Cao Y, Sukawa $Y$, et al. Fusobacterium nucleatum in colorectal carcinoma tissue and patient prognosis. Gut. 2016 Dec;65(12):1973-1980.

18. Piyathilake CJ, Ollberding NJ, Kumar R, Macaluso M, D'Alvarez R, Morrow CD. Cervical microbiota associated with risk of higher grade cervical intraepithelial neoplasia in women infected with high-risk human papillomaviruses. Cancer Prev Res (Phila). 2016;9:357-66.

19. Romano G, Sgambato A, Mancini R, Capelli $G$, et al. 8-hydroxy-20 deoxyguanosine in cervical cells: correlation with grade of dysplasia and human papillomavirus infection. Carcinogenesis. 2000; 21:1143-1147.

20. Scott M, Stites DP, Moscicki AB. Th1 cytokine patterns in cervical human papillomavirus infection. Clin Diagn Lab Immunol. 1999;6:751-755. 